\title{
Multidisciplinary Management of Common Bile Duct Stones
}



Jeffrey W. Hazey

Darwin L. Conwell • Gregory E. Guy

Editors

\section{Multidisciplinary \\ Management \\ of Common Bile \\ Duct Stones}

算 Springer 


\section{Editors}

Jeffrey W. Hazey, MD, FACS

Chief, Division of General and Gastrointestinal Surgery

Department of Surgery

The Ohio State University Wexner Medical Center

Columbus, OH, USA

Gregory E. Guy, MD

Division of Vascular and Interventional Radiology

Department of Radiology

The Ohio State University Wexner Medical Center

Columbus, OH, USA
Darwin L. Conwell, MD

Director, Division of Gastroenterology, Hepatology, and Nutrition

Professor of Medicine

Department of Internal Medicine

The Ohio State University Wexner

Medical Center

Columbus, OH, USA

ISBN 978-3-319-22764-1

DOI 10.1007/978-3-319-22765-8

Library of Congress Control Number: 2015953227

Springer Cham Heidelberg New York Dordrecht London (C) Springer International Publishing Switzerland 2016

This work is subject to copyright. All rights are reserved by the Publisher, whether the whole or part of the material is concerned, specifically the rights of translation, reprinting, reuse of illustrations, recitation, broadcasting, reproduction on microfilms or in any other physical way, and transmission or information storage and retrieval, electronic adaptation, computer software, or by similar or dissimilar methodology now known or hereafter developed.

The use of general descriptive names, registered names, trademarks, service marks, etc. in this publication does not imply, even in the absence of a specific statement, that such names are exempt from the relevant protective laws and regulations and therefore free for general use.

The publisher, the authors and the editors are safe to assume that the advice and information in this book are believed to be true and accurate at the date of publication. Neither the publisher nor the authors or the editors give a warranty, express or implied, with respect to the material contained herein or for any errors or omissions that may have been made.

Printed on acid-free paper

Springer International Publishing AG Switzerland is part of Springer Science+Business Media (www.springer.com) 
I would like to dedicate this textbook to all physicians (surgeons, gastroenterologists, and radiologists) who tirelessly care for patients, often at personal expense, as well as those who have participated in my training throughout the years and have given me the skills and knowledge to effectively treat patients. I would be remiss if I did not thank my colleagues in surgery who teach me something new every day, often commiserating with me after a long or difficult day. All the patients who allow me to operate on them or try novel techniques (sometimes unproven) are the ones who truly deserve thanks. Finally, I wish to thank my wife, Jeannie, and my daughters, Jessica and Jordan, who tolerate my long hours and fatigue in an effort to make a difference. Without their unwavering support, I would not be able to do anything discussed in the text. 



\section{Foreword}

Bile duct stones are not a new problem for surgeons. For decades, a direct open surgical approach to the bile duct was the only definitive therapy for this malady. With the advent of advanced endoscopic, radiologic, and minimally invasive surgical techniques, the treating physician is presented with a myriad of choices and decisions regarding the timing and approach to the stones. Furthermore, the introduction of new surgical procedures for the treatment of gastric disease and morbid obesity often has made access to the biliary tree challenging. In this book, the authors have endeavored to outline each of the techniques for imaging and treating common bile duct stones and to equitably assess the timing and value of each method. It is imperative for practicing surgeons and gastroenterologists to be familiar with the material presented herein in order to provide their patients with the safest and most effective care.

Cleveland, $\mathrm{OH}, \mathrm{USA}$

Jeffrey L. Ponsky 



\section{Preface}

In some large academic medical centers, tradition and culture reign. These "traditions" often lead us to "silos" that exist between specialties, with little cross-fertilization. Often these "turf wars" create a lack of information transfer that ultimately results in inefficient care for patients, and some may argue, less quality care. I truly hope that by outlining a multidisciplinary approach to the management of common bile duct stones, I can educate readers on alternative methods to expand the options available to patients. Collective intelligence will always improve patient care along with increased professionalism between specialties. After all, if all you possess is a hammer, everything begins to look like a nail.

It is with tremendous gratitude I extend to my colleagues and patients who are deserving of the praise that ultimately led to the development of this textbook. As is often the case, this textbook is the work of several professional colleagues who tirelessly care for patients and "fight the good fight" every day, night, and weekend, with little regard for their personal lives. In a similar light, patients consent to sometimes invasive procedures that may put their lives and health at risk in an effort to ameliorate their suffering. It is the patients who allow us to perform sometimes less proven techniques in an effort to advance medical science and improve the quality of health care.

Columbus, OH, USA

Jeffrey W. Hazey 



\section{Contents}

1 Historical Perspective and Treatments

for Common Bile Duct Stones.

Jeffrey W. Hazey

2 Common Bile Duct Stones: Health Care Problem and Incidence.

Joshua S. Winder and Eric M. Pauli

3 Bile Duct Stones: Making the Radiologic Diagnosis

Sunny Jaiswal and Suresh Chamarthi

4 Making the Diagnosis: Gastroenterology

Joshua R. Peck, Nicholas Latchana, Samer El-Dika, and Sheetal Sharma

5 Making the Diagnosis: Surgery, a Rational Approach to the Patient with Suspected CBD Stones

Robert D. Fanelli and Brandon D. Andrew

6 Percutaneous Biliary Access: Considerations, Techniques, and Complications

Bill S. Majdalany and James Spain

7 Special Considerations for the Gastroenterologist

Veeral M. Oza and Marty M. Meyer

8 Special Considerations for the Surgeon

Michael Paul Meara

9 Percutaneous Methods of Common Bile Duct Stone Retrieval

Joshua D. Dowell, Jeffrey Weinstein, Annie Lim, and Gregory E. Guy

10 Gastroenterologic Treatment and Outcomes

Mohammad H. Shakhatreh and J. Royce Groce

11 Surgical Treatment and Outcomes

Ashwini Kumar and Jose M. Martinez

12 Surgical Procedures to Prevent Recurrence

Edward L. Jones 
13 Management of Medical Complications of Gallstone Disease

Victorio Pidlaoan and Somashekar G. Krishna

14 Management of Surgical Complications.

Helmi Khadra, Terence Jackson, and Jeffrey Marks

15 Special Considerations: Management of Common

Bile Duct Stones in the Bariatric Patient

Teresa S. Jones and Vimal K. Narula

16 Management of Choledocholithiasis

in the Cirrhotic Patient.

Eliza W. Beal and Sylvester M. Black

17 Nonprocedural Management of Common

Bile Duct Stones.

Reshi C. Kanuru and Edward Levine

Index 


\section{Contributors}

Brandon D. Andrew, M.D. Department of Surgery, The Guthrie Robert Packer Hospital Residency Program in Surgery, Sayre, PA, USA

Eliza W. Beal, M.D. Department of General Surgery, The Ohio State University Wexner Medical Center, Columbus, OH, USA

Sylvester M. Black, M.D., Ph.D. Department of General Surgery, The Ohio State University Wexner Medical Center, Columbus, OH, USA

Suresh Chamarthi, M.B.B.S. Department of Radiology, The Ohio State University Wexner Medical Center, Columbus, OH, USA

Darwin L. Conwell, M.D. Director, Division of Gastroenterology, Hepatology, and Nutrition, Professor of Medicine, Department of Internal Medicine, The Ohio State University Wexner Medical Center, Columbus, OH, USA

Joshua D. Dowell, M.D., Ph.D. Division of Vascular and Interventional Radiology, Department of Radiology, Wexner Medical Center, The Ohio State University, Columbus, OH, USA

Samer El-Dika, M.D., M.Sc. Division of Gastroenterology, Hepatology, and Nutrition, Department of Internal Medicine, The Ohio State University Wexner Medical Center, Columbus, OH, USA

Section of Advanced Therapeutic Endoscopy, Division of Gastroenterology, The Ohio State University Wexner Medical Center, Columbus, OH, USA

Robert D. Fanelli, M.D., M.H.A., F.A.C.S. Department of Surgery, The Guthrie Clinic, Sayre, PA, USA

Clinical Professor of Surgery, The Commonwealth Medical College, Scranton, PA, USA

Clinical Professor of Surgery, SUNY Upstate Medical University, Binghamton, NY, USA

Professor of Surgery, Albany Medical College, Albany, NY, USA 
J. Royce Groce, M.D. Division of Gastroenterology, Hepatology, and Nutrition, Department of Internal Medicine, The Ohio State University Wexner Medical Clinic, Columbus, OH, USA

Gregory E. Guy, M.D. Division of Vascular and Interventional Radiology, Department of Radiology, The Ohio State University Wexner Medical Center, Columbus, $\mathrm{OH}$, USA

Jeffrey W. Hazey, M.D., F.A.C.S. Chief, Division of General and Gastrointestinal Surgery, Department of Surgery, The Ohio State University Wexner Medical Center, Columbus, OH, USA

Terence Jackson, M.D. Department of Surgery, University Hospitals Case Medical Center, Cleveland, OH, USA

Sunny Jaiswal, M.D., M.B.A. Department of Radiology, The Ohio State University Wexner Medical Center, Columbus, OH, USA

Edward L. Jones, M.D. Department of Surgery, Denver VA Medical Center and the University of Colorado, Denver, CO, USA

Teresa S. Jones, M.D. Department of Surgery, The Ohio State University Wexner Medical Center, Columbus, OH, USA

Reshi C. Kanuru, M.D. Division of Gastroenterology, Hepatology, and Nutrition, Department of Internal Medicine, The Ohio State University Wexner Medical Center, Columbus, OH, USA

Helmi Khadra, M.D. Department of Surgery, University Hospitals Case Medical Center, Cleveland, OH, USA

Somashekar G. Krishna, M.D., M.P.H. Division of Gastroenterology, Hepatology and Nutrition, Department of Internal Medicine, The Ohio State University Wexner Medical Center, Columbus, OH, USA

Ashwini Kumar, M.B.B.S., M.D. Department of Laparoendoscopic Surgery, University of Miami, Miami, FL, USA

Jourdanton, TX, USA

Nicholas Latchana, M.D., M.S. Department of General Surgery, The Ohio State University Wexner Medical Center, Columbus, OH, USA

Edward Levine, M.D. Department of Internal Medicine, The Ohio State University Wexner Medical Center, Columbus, OH, USA

Annie Lim, D.O. Division of Vascular and Interventional Radiology, Department of Radiology, Wexner Medical Center, The Ohio State University, Columbus, $\mathrm{OH}$, USA

Bill S. Majdalany, M.D. Department of Radiology, University of Michigan, Ann Arbor, MI, USA

Jeffrey Marks, M.D. Department of Surgery, University Hospitals Case Medical Center, Cleveland, OH, USA

Jose M. Martinez, M.D. Department of Surgery, Miller School of Medicine, University of Miami, Miami, FL, USA 
Michael Paul Meara, M.D., M.B.A. Division of General and Gastrointestinal Surgery, The Ohio State University Wexner Medical Center, University Hospital East, Columbus, OH, USA

Marty M. Meyer, M.D. Division of Gastroenterology, Hepatology and Nutrition, The Ohio State University Wexner Medical Center, Columbus, $\mathrm{OH}, \mathrm{USA}$

Vimal K. Narula, M.D. Department of Surgery, The Ohio State University Wexner Medical Center, Columbus, OH, USA

Veeral M. Oza, M.D. Division of Gastroenterology, Hepatology and Nutrition, Department of Internal Medicine, The Ohio State University Wexner Medical Center, Columbus, OH, USA

Eric M. Pauli, M.D. Division of Minimally Invasive and Bariatric Surgery, Department of Surgery, Penn State Hershey Medical Center, Hershey, PA, USA

Joshua R. Peck, M.D. Division of Gastroenterology, Hepatology and Nutrition, Department of Internal Medicine, The Ohio State University Wexner Medical Center, Columbus, OH, USA

Victorio Pidlaoan, M.D. Division of Gastroenterology, Hepatology and Nutrition, Department of Internal Medicine, The Ohio State University Wexner Medical Center, Columbus, OH, USA

Mohammad H. Shakhatreh, M.D., M.P.H. Division of Gastroenterology, Hepatology, and Nutrition, Department of Internal Medicine, The Ohio State University Wexner Medical Clinic, Columbus, OH, USA

Sheetal Sharma, M.D. Section of Advanced Therapeutic Endoscopy, Division of Gastroenterology, The Ohio State University Wexner Medical Center, Columbus, $\mathrm{OH}$, USA

James Spain, M.D. Department of Radiology, The Ohio State University Wexner Medical Center, Columbus, OH, USA

Jeffrey Weinstein, M.D. Division of Vascular and Interventional Radiology, Department of Radiology, Einstein Medical Center, Philadelphia, PA, USA

Joshua S. Winder, M.D. Division of Minimally Invasive and Bariatric Surgery, Department of Surgery, Penn State Hershey Medical Center, Hershey, PA, USA 